Pacific

Journal of

Mathematics

MODULE SUPERSINGULIER, FORMULE DE GROSS-KUDLA ET POINTS RATIONNELS DE COURBES MODULAIRES

MARUSiA REBOLLEDO 


\title{
MODULE SUPERSINGULIER, FORMULE DE GROSS-KUDLA ET POINTS RATIONNELS DE COURBES MODULAIRES
}

\author{
MARUSia REBOLLEDO
}

\begin{abstract}
We show how the Gross-Kudla formula about triple product $\boldsymbol{L}$-functions allows us to construct degree-zero elements of the supersingular module annihilated by the winding ideal. Using the method of Parent, we apply those results to the study of rational points on modular curves, determining a set of primes of analytic density $1-9 / 2^{10}$ for which the quotient of $X_{0}\left(p^{r}\right)$ $(r>1)$ by the Atkin-Lehner operator $w_{p^{r}}$ has no rational points other than the cusps and the $C M$ points.
\end{abstract}

\section{Introduction}

Pour $N>0$ un entier, notons $X_{0}(N)$ la courbe modulaire sur $\mathbb{Q}$ classifiant grossièrement les courbes elliptiques généralisées munies d'une $N$-isogénie. La motivation initiale de ces travaux est l'étude des points rationnels du quotient $X_{0}^{+}\left(p^{r}\right)$ de $X_{0}\left(p^{r}\right)$ par l'involution d'Atkin-Lehner $w_{p^{r}}$ pour $p$ un nombre premier et $r>1$ un entier. Pour cela, nous reprenons une méthode de Parent [2005] s'inspirant des travaux de Momose [1984; 1986; 1987] et faisant appel au module supersingulier.

Fixons $p>3$ un nombre premier et $\overline{\mathbb{F}}_{p}$ une clôture algébrique de $\mathbb{F}_{p}$. Nous appelons module supersingulier le $\mathbb{Z}$-module libre $\mathscr{P}$ engendré par l'ensemble fini $\mathscr{S}=\left\{x_{0}, \ldots, x_{g}\right\}$ des classes d'isomorphismes de courbes elliptiques supersingulières sur $\overline{\mathbb{F}}_{p}$. Notons $\mathscr{P}^{0}$ le sous-groupe de $\mathscr{P}$ constitué des éléments de degré nul. On peut munir $\mathscr{P}^{0}$ d'une action de l'anneau $\mathbb{T}$ engendré par les opérateurs de Hecke agissant sur l'espace vectoriel $\mathrm{S}_{2}\left(\Gamma_{0}(p)\right)$ des formes paraboliques de poids 2 pour $\Gamma_{0}(p)$ (voir 1A). Notons $\mathscr{P}^{0}\left[I_{e}\right]$ l'ensemble des éléments de $\mathscr{P}^{0}$ annulés par l'idéal d'enroulement $I_{e} \subset \mathbb{T}$ c'est-à-dire l'annulateur des formes primitives $f \in \mathrm{S}_{2}\left(\Gamma_{0}(p)\right)$ telles que $L(f, 1) \neq 0$. Pour $j \in \mathbb{P}^{1}\left(\overline{\mathbb{F}}_{p}\right)$ un invariant non supersingulier, considérons l'application

$$
\begin{aligned}
\iota_{j}: \quad & \overline{\mathbb{F}}_{p} \\
\sum_{i=0}^{g} \lambda_{i} x_{i} & \mapsto \sum_{i=0}^{g} \frac{\lambda_{i}}{j-j_{i}}
\end{aligned}
$$

\footnotetext{
MSC2000: primary 14G05, 11G05, 11G18; secondary 14G10, 11R52.
}

Mots-clefs: rational points on modular curves, supersingular module, special values of $L$-functions. 
où, pour $i \in\{0, \ldots, g\}, j_{i}$ est l'invariant d'une courbe elliptique $E_{i} \in x_{i}$. Parent a mis en évidence le critère suivant (voir propositions 3.1 et 3.2 de [Parent 2005]) :

(C) Soit $p \geq 11$. Supposons que pour tout $j \in \mathbb{F}_{p}$ non supersingulier, il existe $x \in \mathscr{P}^{0}\left[I_{e}\right]$ tel que $\iota_{j}(x) \neq 0$. Alors $X_{0}^{+}\left(p^{r}\right)(\mathbb{Q})$ est trivial c'est-àdire ne contient que des pointes et des points $C M$.

Pour $A$ un anneau et $f: M \rightarrow N$ un homomorphisme de $\mathbb{Z}$-modules, on note $M_{A}=M \otimes A$ et on note encore $f: M_{A} \rightarrow N_{A}$ l'homomorphisme de $A$-modules obtenu par extension des scalaires. Soient $n$ le numérateur de $(p-1) / 12$ et $\pi^{0}$ : $\mathscr{P}_{\mathbb{Z}[1 / n]} \rightarrow \mathscr{P}_{\mathbb{Z}[1 / n]}^{0}$ la projection orthogonale (voir Section 1C). Pour $D>0$ notons $\gamma_{D} \in \mathscr{P}_{\mathbb{Q}}$ le $D$-ième élément de Gross (voir $3 \mathrm{~A}$ ). Notons $\operatorname{Disc}(p)$ l'ensemble des discriminants quadratiques imaginaires ${ }^{1}$ premiers à $p$. Parent déduit de la formule de Gross-Zhang que $\gamma_{D}^{0}:=\pi^{0}\left(\gamma_{D}\right) \in \mathscr{P}^{0}\left[I_{e}\right]_{\mathbb{Q}}$ pour $-D \in \operatorname{Disc}(p)$. Considérons une autre famille d'éléments du module supersingulier :

$$
y_{m}=\sum_{i=0}^{g}\left\langle T_{m} x_{i}, \frac{x_{i}}{w_{i}}\right\rangle x_{i} \in \mathscr{P} \quad \text { et } \quad y_{m}^{0}=\pi^{0}\left(y_{m}\right) \in \mathscr{P}_{\mathbb{Z}[1 / n]}^{0} \quad(m \geq 1)
$$

où $\langle$,$\rangle est l'accouplement bilinéaire sur \mathscr{P}$ défini en (3) et $w_{i}=\left|\operatorname{Aut}\left(E_{i}\right)\right| / 2$ pour $E_{i} \in x_{i}$.

Théorème 0.1. Pour tout entier $m \geq 1, y_{m}^{0} \in \mathscr{P}^{0}\left[I_{e}\right]_{\mathbb{Q}}$.

Notons $a_{E}=\sum_{i=0}^{g} x_{i} / w_{i}$ l'élément d'Eisenstein (voir le Section 1C). L'assertion précédente peut se déduire de la formule de Gross-Kudla ou, pour certaines valeurs de $m$, de la formule de Gross-Zhang et de la proposition suivante

Proposition 0.2. On $a$

$$
y_{m}=\epsilon(m) a_{E}+\sum_{\substack{(s, d) \in \mathbb{Z}^{2} \\ 4 m-s^{2}=d r^{2}>0}} \gamma_{d} \quad(m \geq 1)
$$

où $\epsilon(m)=1$ si $m$ est un carré et $\epsilon(m)=0$ sinon.

La démonstration de la proposition 0.2 s'inspire du calcul classique qui permet d'établir la formule des traces d'Eichler (voir [Eichler 1955], [Gross 1987] et la Section 3B).

Parent [2005] montre que $\left\{\gamma_{D}^{0},-D \in \operatorname{Disc}(p)\right\}$ engendre le $\mathbb{Q}$-espace vectoriel $\mathscr{P}^{0}\left[I_{e}\right]_{\mathbb{Q}}$. Nous montrons au Section 3B les propositions suivantes :

Proposition 0.3. Le $\mathbb{Q}$-espace vectoriel engendré par $\left(y_{m}^{0}\right)_{m \geq 1}$ est égal au $\mathbb{Q}$ espace vectoriel engendré par $\left(y_{m}^{0}\right)_{1 \leq m \leq g+1}$.

\footnotetext{
${ }^{1}$ On appelle ici discriminant quadratique imaginaire le discriminant d'un ordre d'un corps quadratique imaginaire. C'est donc un carré mutiplié par un discriminant fondamental.
} 
Proposition 0.4. Le $\mathbb{T}_{\mathbb{Q}}$-module engendré par $\left\{y_{m}^{0}, m \geq 1\right\}$ est égal à $\mathscr{P}^{0}\left[I_{e}\right]_{\mathbb{Q}}$.

Pour $-d<0$ un discriminant fondamental, notons $\varepsilon_{d}$ le caractère non trivial de $\operatorname{Gal}(\mathbb{Q}(\sqrt{-d}) / \mathbb{Q})$. Les propositions $0.2,0.3$ et 0.4 entraînent une version précise d'un théorème de non-annulation :

Corollaire 0.5. Si $f$ est une forme primitive de poids 2 pour $\Gamma_{0}(p)$ telle que $L(f, 1) \neq 0$, alors il existe $d \leq 4 g+4$ tel que $L\left(f \otimes \varepsilon_{d}, 1\right) \neq 0$.

Pour $(m, p)=1, y_{m}$ énumère les boucles du graphe des $m$-isogénies étudié par Mestre et Oesterlé [ $\geq 2008$ ]. Cela permet de faire les calculs (Section 4B) conduisant au Théorème 0.6 suivant.

Considérons le nombre premier $p_{0}=45321935159$. Soit $\mathscr{C}$ l'ensemble des nombres premiers $p$ qui sont un carré modulo 3,4 et 7 et tels que l'une des conditions suivantes soit vérifiée :

(1) $p$ carré modulo $5,11,19,23,43,67,163$, non carré modulo 8 ;

(2) $p$ carré modulo $8,11,19$, et modulo au moins deux des nombres premiers 43, 67, 163, et vérifiant l'une des conditions suivantes

(a) $p$ carré modulo 5;

(b) $p$ non carré modulo 5 et 23 ;

(c) $p$ non carré modulo 5 et carré modulo 23, 59, 71;

(d) $p$ non carré modulo 5, 59, 71 et carré modulo 23;

(3) $p$ carré modulo 5, 8, 11, 43, 67, 163, non carré modulo 19 et l'une des conditions suivantes est vérifiée :

(a) $p$ carré modulo 23 ;

(b) $p$ non carré modulo 23 et $\left(\frac{p}{31}\right)\left(\frac{p}{36319}\right)\left(\frac{p}{p_{0}}\right)=1$.

(4) $p$ carré modulo $5,8,19,43,67,163$, non carré modulo 11 et $p$ carré modulo au moins un des nombres : 23, 797.

Théorème 0.6. Si $p \geq 11, p \neq 13$ et $p \notin \mathscr{C}$, alors $X_{0}^{+}\left(p^{r}\right)(\mathbb{Q})$ est trivial.

L'ensemble $\mathscr{C}$ est de densité analytique $9 / 2^{10}$. Parent [2005] avait obtenu un résultat analogue avec une densité de $7 / 2^{9}$. Le cas $r=2$ de ce théorème constitue une avancée en direction du cas normalisateur d'un Cartan déployé d'un problème de Serre sur la torsion des courbes elliptiques. (Pour un énoncé de ce problème, se reporter à [Serre 1972; Serre 1986, p. 288].) La liste des nombres premiers $11 \leq p \leq 50000, p \neq 13$ dans $\mathscr{C}$ est donnée dans la Table 3 (page 182). 


\section{Préliminaires sur le module supersingulier}

1A. Réalisations géométriques et opérateurs de Hecke. Soit $\widetilde{\mathbb{T}}$ l'anneau engendré par l'action des opérateurs de Hecke $T_{m}, m \geq 1$ sur le $\mathbb{C}$-espace vectoriel $\mathrm{M}_{2}\left(\Gamma_{0}(p)\right)$ des formes modulaires de poids 2 pour $\Gamma_{0}(p)$. Cette action se factorise par $\mathbb{T}$ sur $\mathrm{S}_{2}\left(\Gamma_{0}(p)\right)$. Soient $X_{0}(p)_{\mathbb{Z}}$ la normalisation de $\mathbb{P}_{\mathbb{Z}}^{1}$ dans $X_{0}(p)$ via le morphisme composé $X_{0}(p) \rightarrow X_{0}(1) \cong \mathbb{P}_{\mathbb{Q}}^{1}$. La fibre $X_{0}(p)_{\mathbb{F}_{p}}$ de $X_{0}(p)_{\mathbb{Z}}$ en $p$ est constituée de deux copies de $\mathbb{P}_{\mathbb{F}_{p}}^{1}$ qui sont échangées par l'opérateur d'Atkin-Lehner $w_{p}$ et se coupent transversalement. Les points doubles de $X_{0}(p)_{\mathbb{F}_{p}}$ sont en correspondance bijective avec les classes $x_{0}, \ldots, x_{g}$ de $\mathscr{S}$ et $g$ n'est autre que le genre de $X_{0}(p)$. Notons $J_{0}(p)$ la jacobienne de $X_{0}(p)$ et $\widetilde{J}$ la jacobienne généralisée de $X_{0}(p)$ relativement aux pointes. L'anneau $\widetilde{\mathbb{T}}($ resp. $\mathbb{T}$ ) est isomorphe à l'anneau engendré par les endomorphismes de $\widetilde{J}$ (resp. $J_{0}(p)$ ) provenant des correspondances de Hecke sur $X_{0}(p)$. Soient $J_{0}(p)_{\mathbb{Z}}$ et $\widetilde{J}_{\mathbb{Z}}$ les modèles de Néron respectifs de $J_{0}(p)$ et $\widetilde{J}$ sur $\mathbb{Z}$. Le groupe $\mathscr{P}=\mathbb{Z}[\mathscr{Y}]$ (resp. $\mathscr{P}^{0}$ ) s'identifie au groupe des caractères de la composante neutre de la fibre en $p$ de $\widetilde{J}_{\mathbb{Z}}$ (resp. $J_{0}(p)_{\mathbb{Z}}$ ) (voir [Raynaud 1991] et [de Shalit 1995, 2.3]). Cela définit par transport de structure une action de $\widetilde{\mathbb{T}}$ sur $\mathscr{P}$ qui laisse stable $\mathscr{P}^{0}$ et se factorise par $\mathbb{T}$ sur $\mathscr{P}^{0}$. L'action de $\widetilde{\mathbb{T}}$ sur la classe d'isomorphisme $[E]$ d'une courbe elliptique $E$ supersingulière sur $\overline{\mathbb{F}}_{p}$ est donnée par $T_{m}([E])=\sum_{C}[E / C](m \geq 1)$, où $C$ parcourt l'ensemble des sous-schémas en groupes finis d'ordre $m$ de $E$ (voir [Raynaud 1991] ou [Mestre et Oesterlé $\geq 2008$, 1.2.1]). Sur la base $\left(x_{i}\right)_{0 \leq i \leq g}$ de $\mathscr{P}$, l'action de $T_{m}(m \geq 1)$ est donnée par la transposée de la matrice d'Eichler-Brandt $B(m)=\left(B_{i, j}(m)\right)_{0 \leq i, j \leq g}$ (voir [Gross 1987, sections 1 et 4$]$ ).

1B. Accouplement bilinéaire. Soit $\delta$ le dénominateur de $(p-1) / 12$. Rappelons que $w_{i}=\left|\operatorname{Aut}\left(E_{i}\right)\right| / 2\left(i \in\{0, \ldots, g\}, E_{i} \in x_{i}\right)$ vérifient

$$
\prod_{i=0}^{g} w_{i}=\delta \quad \text { et } \quad \sum_{i=0}^{g} 1 / w_{i}=(p-1) / 12 .
$$

Le $\mathbb{Z}$-module $\mathscr{P}$ est muni de l'accouplement bilinéaire non dégénéré $\langle$,$\rangle :$ $\mathscr{P} \times \mathscr{P} \rightarrow \mathbb{Z}$ défini par

$$
\left\langle x_{i}, x_{j}\right\rangle=w_{i} \delta_{i, j} \quad(0 \leq i, j \leq g)
$$

où $\delta_{i, j}$ est le symbole de Krönecker. Les opérateurs de Hecke sont auto-adjoints pour $\langle$,$\rangle . Cet accouplement induit un homomorphisme injectif de \widetilde{\mathbb{T}}$-modules de $\mathscr{P}$ dans $\check{\mathscr{P}}=\operatorname{Hom}(\mathscr{P}, \mathbb{Z})$ (sur lequel $\tilde{\mathbb{T}}$ agit par dualité) de conoyau isomorphe à $\mathbb{Z} / \delta \mathbb{Z}$ identifiant $\check{\mathscr{P}}$ au sous- $\widetilde{\mathbb{T}}$-module

$$
\bigoplus_{i=0}^{g} \mathbb{Z} \frac{x_{i}}{w_{i}}
$$


de $\mathscr{P}_{\mathbb{Q}}$ (voir [Gross 1987] ou [Emerton 2002, lemme 3.16]). L'accouplement canonique $\mathscr{P} \times \check{\mathscr{P}} \rightarrow \mathbb{Z}$ étend donc l'accouplement $\langle$,$\rangle et sera encore noté \langle$,$\rangle .$ L'homomorphisme injectif de $\mathbb{T}$-modules de $\mathscr{P}^{0}$ dans $\mathscr{\mathscr { P }}^{0}=\operatorname{Hom}\left(\mathscr{P}^{0}, \mathbb{Z}\right)$ induit par $\langle$,$\rangle est de conoyau isomorphe à \mathbb{Z} / n \mathbb{Z}$ (loc. cit.). Sur $\mathscr{\mathscr { P }}^{0} / \mathscr{P}^{0}$ qui s'identifie au groupe des composantes de la fibre en $p$ de $J_{0}(p)_{\mathbb{Z}}$, l'accouplement $\langle$,$\rangle n'est$ autre que l'accouplement de monodromie (voir [Illusie 1991] ou l'appendice de [Bertolini et Darmon 1997]).

Notons $\sum_{m \geq 0} a_{m}(f) q^{m}$ le développement de Fourier à l'infini de $f \in \mathrm{M}_{2}\left(\Gamma_{0}(p)\right)$. Considérons le $\mathbb{Z}$-module $\mathcal{M}$ des formes modulaires $f$ telles que $a_{0}(f) \in \mathbb{Q}$ et $a_{m}(f) \in \mathbb{Z}$ pour $m \geq 1$ et $\mathcal{M}^{0}$ le sous- $\mathbb{Z}$-module de $\mathcal{M}$ constitué des formes paraboliques $^{2}$. L'action de $\widetilde{\mathbb{T}}$ sur $\mathrm{M}_{2}\left(\Gamma_{0}(p)\right)$ laisse stables $\mu$ et $M^{0}$ et se factorise par $\mathbb{T}$ sur $M^{0}$. On a $\mu_{\mathbb{C}}=\mathrm{M}_{2}\left(\Gamma_{0}(p)\right)$ et $M_{\mathbb{C}}^{0}=\mathrm{S}_{2}\left(\Gamma_{0}(p)\right)$. L'accouplement sur $M \times \widetilde{\mathbb{T}}$ défini par $(f, T) \mapsto a_{1}(f \mid T)$ induit un isomorphisme de $\widetilde{\mathbb{T}}$-modules (resp. de $\mathbb{T}$-modules)

$$
\mu \stackrel{\sim}{\rightarrow} \operatorname{Hom}(\tilde{\mathbb{T}}, \mathbb{Z}) \quad\left(\operatorname{resp} . \mathcal{M}^{0} \stackrel{\sim}{\rightarrow} \operatorname{Hom}(\mathbb{T}, \mathbb{Z})\right)
$$

(voir [Ribet 1983, théorème 2.2 ; Emerton 2002, proposition 1.3]). Les homomorphismes de $\widetilde{\mathbb{T}}$-modules et $\mathbb{T}$-modules

$$
\begin{aligned}
\theta: \mathscr{P} \otimes_{\widetilde{\mathbb{T}}} \check{\mathscr{P}} & \rightarrow \operatorname{Hom}(\widetilde{\mathbb{T}}, \mathbb{Z}) \cong \mu \\
x \otimes_{\widetilde{\mathbb{U}}} y & \mapsto \frac{1}{2}(\operatorname{deg} x \cdot \operatorname{deg} y)+\sum_{m \geq 1}\left\langle T_{m} x, y\right\rangle q^{m}
\end{aligned}
$$

et

$$
\theta^{0}: \mathscr{P}^{0} \otimes_{\mathbb{T}} \check{\mathscr{P}}^{0} \rightarrow \operatorname{Hom}(\mathbb{T}, \mathbb{Z}) \cong M^{0}
$$

qui se déduisent de l'accouplement $\langle$, $\rangle$, sont des surjections (voir [Emerton 2002, théorème 0.10]).

1C. Le $\tilde{\mathbb{T}}$-module $\mathscr{P}$. Les $\widetilde{\mathbb{T}}_{\mathbb{Q}}$-modules $\mathscr{P}_{\mathbb{Q}}$ et $\mu_{\mathbb{Q}}$ et les $\mathbb{T}_{\mathbb{Q}}$-modules $\mathscr{P}_{\mathbb{Q}}^{0}$ et $\mu_{\mathbb{Q}}^{0}$ sont libres de rang 1 (voir par exemple [Gross 1987] et [Miyake 1989]). Appelons forme de Hecke une forme modulaire de poids 2 pour $\Gamma_{0}(p)$ propre pour tous les opérateurs de Hecke et normalisée, et forme primitive une forme de Hecke parabolique. On note Prim l'ensemble des formes primitives. Les idempotents primitifs de $\widetilde{\mathbb{T}}_{\bar{Q}}$ sont en correspondance bijective avec les formes de Hecke et engendrent les sous- $\widetilde{\mathbb{T}}_{\overline{\mathbb{Q}}}$-modules irréductibles de $\widetilde{\mathbb{T}}_{\overline{\mathbb{Q}}}$. On note $\mathbf{1}_{f}$ l'idempotent primitif associé à une forme de Hecke $f$. Les opérateurs de Hecke étant autoadjoints pour $\langle$,$\rangle , le$ $\widetilde{\mathbb{T}}_{\overline{\mathbb{Q}}}$-module $\mathscr{P}_{\overline{\mathbb{Q}}}$ se décompose en somme directe de sous-espaces propres orthogonaux. Pour $f$ une forme primitive, le sous-espace $\mathscr{P}_{\overline{\mathbb{Q}}}^{f}=\mathbf{1}_{f}\left(\mathscr{P}_{\overline{\mathbb{Q}}}\right)$ est une $\overline{\mathbb{Q}}$-droite dont on choisit un vecteur directeur $a_{f}$. Notons $\sigma^{\prime}(m)$ la somme des diviseurs de

\footnotetext{
${ }^{2}$ Pour $f \in \mathcal{M}$, on a en fait $\delta a_{0}(f) \in \mathbb{Z}$ (voir [Emerton 2002, proposition 1.1]). Attention, les notations diffèrent de celles adoptées dans [Emerton 2002] où $\mathcal{M}$ est noté $\mathcal{N}$.
} 
$m$ premiers à $p$. Le sous-espace propre associé à la série d'Eisenstein normalisée $E=(p-1) / 24+\sum_{m \geq 1} \sigma^{\prime}(m) q^{m}$ est engendré par l'élément d'Eisenstein

$$
a_{E}=\sum_{i=0}^{g} \frac{x_{i}}{w_{i}} \in \check{\mathscr{P}} \subset \mathscr{P}_{\mathbb{Z}[1 / \delta]} .
$$

De plus, $a_{E}$ vérifie $\left\langle x, a_{E}\right\rangle=\operatorname{deg} x(x \in \mathscr{P})$. Par conséquent, $\mathscr{P}^{E}:=\mathbb{Z} . a_{E}$ est le $\mathbb{Z}$-module orthogonal à $\mathscr{P}^{0}$ pour $\langle\rangle:, \mathscr{P} \times \mathscr{\mathscr { P }} \rightarrow \mathbb{Z}$. On note

$$
\begin{aligned}
\pi^{0}: \mathscr{P}_{\mathbb{Z}[1 / n]} & \rightarrow \mathscr{P}_{\mathbb{Z}[1 / n]}^{0} \\
x & \mapsto x-\frac{12}{p-1} \operatorname{deg}(x) a_{E}
\end{aligned}
$$

la projection orthogonale.

1D. Produits tensoriels. Considérons le $\widetilde{\mathbb{T}}$-module $\mathscr{P} \otimes_{\widetilde{\pi}} \mathscr{P}$. Les sous-espaces $\widetilde{\mathbb{T}}_{\overline{\mathbb{Q}}^{-}}$

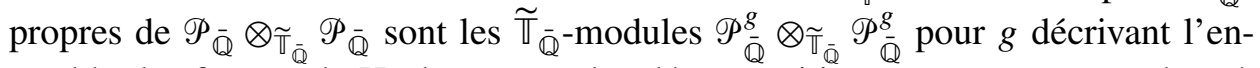
semble des formes de Hecke et on a les décompositions en sous-espaces deux à deux orthogonaux

$\mathscr{P}_{\mathbb{Q}} \otimes_{\widetilde{\mathbb{T}}_{\mathbb{Q}}} \mathscr{P}_{\mathbb{Q}}=\left(\mathscr{P}_{\mathbb{Q}}^{E} \otimes_{\widetilde{\mathbb{T}}_{\mathbb{Q}}} \mathscr{P}_{\mathbb{Q}}^{E}\right) \oplus\left(\mathscr{P}_{\mathbb{Q}}^{0} \otimes_{\mathbb{T}_{\mathbb{Q}}} \mathscr{P}_{\mathbb{Q}}^{0}\right)$ et $\mathscr{P}_{\overline{\mathbb{Q}}}^{0} \otimes_{\mathbb{T}_{\overline{\mathbb{Q}}}} \mathscr{P}_{\overline{\mathbb{Q}}}^{0}=\bigoplus_{g \in \operatorname{Prim}}\left(\mathscr{P}_{\overline{\mathbb{Q}}}^{g} \otimes_{\mathbb{T}_{\overline{\mathbb{Q}}}} \mathscr{P}_{\overline{\mathbb{Q}}}^{g}\right)$.

En effet, pour toutes formes de Hecke $f, g$ et $h$, on a $\mathbf{1}_{f}\left(\mathscr{P}_{\overline{\mathbb{Q}}}^{g} \otimes_{\widetilde{\mathbb{T}}_{\overline{\mathbb{Q}}}} \mathscr{P}_{\overline{\mathbb{Q}}}^{h}\right) \neq 0$ si et seulement si $f=g=h$. Donc si $g \neq h$, on a $\mathscr{P}_{\overline{\mathbb{Q}}}^{g} \otimes_{\widetilde{\pi}_{\overline{\mathbb{Q}}}} \mathscr{P}_{\overline{\mathbb{Q}}}^{h}=\sum_{f} \mathbf{1}_{f}\left(\mathscr{P}_{\overline{\mathbb{Q}}}^{g} \otimes_{\widetilde{\pi}_{\overline{\mathbb{Q}}}} \mathscr{P}_{\overline{\mathbb{Q}}}^{h}\right)=0$.

Considérons à présent les $\tilde{T}^{\otimes 3}$-modules $\mathscr{P}^{\otimes 3}, \mathcal{M}^{\otimes 3}$, et les $\mathbb{T}^{\otimes 3}$-modules $\mathscr{P}^{0^{\otimes 3}}$ et $\mu^{0^{\otimes 3}}$. Par fonctorialité des algèbres tensorielles, on déduit de l'accouplement $\langle$,$\rangle un accouplement { }^{3}\langle,\rangle^{\otimes 3}: \mathscr{P}^{\otimes 3} \times \check{\mathscr{P}} \otimes 3 \rightarrow \mathbb{Z}$. De même, le produit scalaire de Petersson ( , ) sur $\mu_{\mathbb{C}}^{0} \times M_{\mathbb{C}}$ (normalisé comme dans [Gross 1987] (7.1)) définit le produit scalaire de Petersson $(,)^{\otimes 3}$ sur $\mathcal{M}_{\mathbb{C}}^{0 \otimes 3} \times \mathcal{M}_{\mathbb{C}}^{\otimes 3}$ (normalisé par [Gross et Kudla 1992] (11.3)).

Les $\tilde{\mathbb{T}}_{\mathbb{Q}}^{\otimes 3}$-modules $\mu_{\mathbb{Q}}^{\otimes 3}$ et $\mathscr{P}_{\mathbb{Q}}^{\otimes 3}$ et les $\mathbb{T}_{\mathbb{Q}}^{\otimes 3}$-modules $\mu_{\mathbb{Q}}^{0 \otimes 3}$ et $\mathscr{P}_{\mathbb{Q}}^{0}{ }^{\otimes 3}$ sont libres de rang 1. Les opérateurs de Hecke triples de $\widetilde{\mathbb{T}}^{\otimes 3}$ sont autoadjoints pour $(,)^{\otimes 3}$ et $\langle,\rangle^{\otimes 3}$. Les idempotents primitifs de $\widetilde{\mathbb{T}} \overline{\mathbb{Q}}^{\otimes 3}$ sont de la forme $\mathbf{1}_{F}=\mathbf{1}_{f_{1}} \otimes \mathbf{1}_{f_{2}} \otimes \mathbf{1}_{f_{3}}$ pour $F=f_{1} \otimes f_{2} \otimes f_{3}$ parcourant l'ensemble des formes de Hecke triples (c'està-dire telles que $f_{1}, f_{2}$ et $f_{3}$ soient des formes de Hecke). Le $\overline{\mathbb{Q}}$-espace vectoriel $\mathscr{P} \overline{\mathbb{Q}}=\mathbf{1}_{F} \mathscr{P}_{\overline{\mathbb{Q}}}^{\otimes 3}$ est une $\overline{\mathbb{Q}}$-droite de vecteur directeur $A_{F}=a_{f_{1}} \otimes_{\overline{\mathbb{Q}}} a_{f_{2}} \otimes_{\overline{\mathbb{Q}}} a_{f_{3}}$. On a les décompositions en sous-espaces propres deux à deux orthogonaux

$$
\mathscr{P}_{\overline{\mathbb{Q}}}^{\otimes 3}=\bigoplus_{F} \mathscr{P}_{\overline{\mathbb{Q}}}^{F} \quad \text { et } \quad\left(\mathscr{P}_{\overline{\mathbb{Q}}}^{0}\right)^{\otimes 3}=\bigoplus_{F} \mathscr{P}_{\overline{\mathbb{Q}}}^{F},
$$

\footnotetext{
${ }^{3}$ donné par $\left\langle a_{1} \otimes a_{2} \otimes a_{3}, b_{1} \otimes b_{2} \otimes b_{3}\right\rangle^{\otimes 3}=\prod_{i=1}^{3}\left\langle a_{i}, b_{i}\right\rangle$
} 
la somme directe portant respectivement sur l'ensemble des formes de Hecke triples et l'ensemble des formes primitives triples. On vérifie aisément que pour toute forme de Hecke triple $F$, on a

$$
\mathbf{1}_{F} X=\frac{\left\langle A_{F}, X\right\rangle^{\otimes 3}}{\left\langle A_{F}, A_{F}\right\rangle^{\otimes 3}} A_{F} \quad\left(X \in \mathscr{P}_{\overline{\mathbb{Q}}}^{\otimes 3}\right) .
$$

\section{Formule de Gross-Kudla et éléments de $\mathscr{P}^{0}\left[I_{e}\right]$}

Soit

$$
\Delta_{3}=\sum_{i=0}^{g} \frac{1}{w_{i}} x_{i}^{\otimes 3} \in \mathscr{P}_{\mathbb{Q}}^{\otimes 3}
$$

l'élément diagonal de Gross-Kudla. Posons $s: \mathscr{P} \otimes \mathscr{P} \rightarrow \mathscr{P} \otimes \tilde{\pi} \mathscr{P}$ la surjection canonique,

$$
\bar{\Delta}_{3}=\left(1 \otimes_{\mathbb{Q}} s\right)\left(\Delta_{3}\right) \in \mathscr{P}_{\mathbb{Q}} \otimes_{\mathbb{Q}}\left(\mathscr{P}_{\mathbb{Q}} \otimes_{\widetilde{\pi}_{\mathbb{Q}}} \mathscr{P}_{\mathbb{Q}}\right)
$$

et

$$
\bar{\Delta}_{3}^{0}=\left(\pi^{0} \otimes_{\mathbb{Q}} s\right)\left(\Delta_{3}\right)=\left(\pi^{0} \otimes_{\mathbb{Q}} 1\right)\left(\bar{\Delta}_{3}\right) \in \mathscr{P}_{\mathbb{Q}}^{0} \otimes_{\mathbb{Q}}\left(\mathscr{P}_{\mathbb{Q}} \otimes_{\widetilde{\mathbb{T}}_{\mathbb{Q}}} \mathscr{P}_{\mathbb{Q}}\right) .
$$

Gross et Kudla [1992, théorème 11.1] ont montré que pour $F$ une forme primitive triple, on a

$$
L(F, 2)=\frac{(F, F)^{\otimes 3}}{4 \pi p}\left\langle\mathbf{1}_{F} \Delta_{3}, \mathbf{1}_{F} \Delta_{3}\right\rangle^{\otimes 3} .
$$

En particulier, lorsque $F=f \otimes h \otimes h(f, h \in$ Prim), par [Gross et Kudla 1992, (11.7)], on obtient

$$
L(f, 1) L\left(f \otimes \operatorname{Sym}^{2} h, 2\right)=\frac{(F, F)^{\otimes 3}}{4 \pi p}\left\langle\mathbf{1}_{F} \Delta_{3}, \mathbf{1}_{F} \Delta_{3}\right\rangle^{\otimes 3} .
$$

Nous allons voir que cela entraîne le

Théorème 2.1. On $a$

et

$$
\bar{\Delta}_{3}^{0}=\bar{\Delta}_{3}-\frac{12}{p-1} a_{E} \otimes_{\mathbb{Q}}\left(\sum_{i=0}^{g} x_{i} \otimes_{\widetilde{\pi}_{\mathbb{Q}}} \frac{x_{i}}{w_{i}}\right)
$$

$$
\bar{\Delta}_{3}^{0} \in \mathscr{P}^{0}\left[I_{e}\right]_{\mathbb{Q}} \otimes\left(\mathscr{P}_{\mathbb{Q}}^{0} \otimes_{\mathbb{T}_{\mathbb{Q}}} \mathscr{P}_{\mathbb{Q}}^{0}\right) .
$$

Remarque 2.2. Le $\mathbb{T}$-module $\mathscr{P}^{0}$ est localement libre après extension des scalaires à $\mathbb{Z}$ [1/2] (voir l'introduction de [Emerton 2002]), par conséquent il y a au pire de la 2-torsion dans $\mathscr{P}^{0}\left[I_{e}\right] \otimes\left(\mathscr{P}^{0} \otimes_{\mathbb{T}} \mathscr{P}^{0}\right)$. Ainsi, puisque $\delta \Delta_{3} \in \mathscr{P}^{\otimes 3}$, on a même $n \delta \bar{\Delta}_{3}^{0} \in \mathscr{P}^{0}\left[I_{e}\right] \otimes\left(\mathscr{P}^{0} \otimes \mathbb{T} \mathscr{P}^{0}\right)$ à un élément de 2-torsion près.

Démonstration. On a

$$
\bar{\Delta}_{3}^{0}=\sum_{i=0}^{g}\left(x_{i}-\frac{12}{p-1} a_{E}\right) \otimes_{\mathbb{Q}}\left(x_{i} \otimes_{\tilde{\mathbb{T}}_{\mathbb{Q}}} \frac{x_{i}}{w_{i}}\right),
$$


ce qui prouve la première assertion du Théorème 2.1.

On a dans $\mathscr{P}_{\overline{\mathbb{Q}}}^{0} \otimes_{\overline{\mathbb{Q}}}\left(\mathscr{P}_{\overline{\mathbb{Q}}} \otimes_{\tilde{\mathbb{T}}_{\overline{\mathbb{Q}}}} \mathscr{P}_{\overline{\mathbb{Q}}}\right)$

$$
\begin{aligned}
\bar{\Delta}_{3}^{0} & =\sum_{f, h \in \text { Prim }}\left(\mathbf{1}_{f} \otimes \mathbf{1}_{h}\right)\left(\bar{\Delta}_{3}\right)+\sum_{f \in \text { Prim }}\left(\mathbf{1}_{f} \otimes \mathbf{1}_{E}\right)\left(\bar{\Delta}_{3}\right) \\
& =(1 \otimes s)\left(\sum_{f, h \in \text { Prim }}\left(\mathbf{1}_{f} \otimes \mathbf{1}_{h} \otimes \mathbf{1}_{h}\right)\left(\Delta_{3}\right)+\sum_{f \in \text { Prim }}\left(\mathbf{1}_{f} \otimes \mathbf{1}_{E} \otimes \mathbf{1}_{E}\right)\left(\Delta_{3}\right)\right) .
\end{aligned}
$$

Pour tout $f \in$ Prim, on a (voir (4))

$$
\left(\mathbf{1}_{f} \otimes \mathbf{1}_{E} \otimes \mathbf{1}_{E}\right)\left(\Delta_{3}\right)=\frac{\left\langle\Delta_{3}, a_{f} \otimes a_{E} \otimes a_{E}\right\rangle^{\otimes 3}}{\left\langle a_{f} \otimes a_{E} \otimes a_{E}, a_{f} \otimes a_{E} \otimes a_{E}\right\rangle^{\otimes 3}}\left(a_{f} \otimes a_{E} \otimes a_{E}\right) .
$$

$\operatorname{Or}\left\langle\Delta_{3}, a_{f} \otimes a_{E} \otimes a_{E}\right\rangle^{\otimes 3}=\sum_{i=0}^{g} \frac{1}{w_{i}}\left\langle x_{i}, a_{f}\right\rangle\left\langle x_{i}, a_{E}\right\rangle^{2}=\left\langle a_{E}, a_{f}\right\rangle=0$. Par conséquent,

$$
\begin{aligned}
\bar{\Delta}_{3}^{0} & =(1 \otimes s)\left(\sum_{f, h \in \text { Prim }}\left(\mathbf{1}_{f} \otimes \mathbf{1}_{h} \otimes \mathbf{1}_{h}\right)\left(\Delta_{3}\right)\right) \\
& =(1 \otimes s)\left(\sum_{\substack{f, h \in \text { Prim } \\
L(f, 1) \neq 0}}\left(\mathbf{1}_{f} \otimes \mathbf{1}_{h} \otimes \mathbf{1}_{h}\right)\left(\Delta_{3}\right)+\sum_{\substack{f, h \in \text { Prim } \\
L(f, 1)=0}}\left(\mathbf{1}_{f} \otimes \mathbf{1}_{h} \otimes \mathbf{1}_{h}\right)\left(\Delta_{3}\right)\right) .
\end{aligned}
$$

Puisque $(,)^{\otimes 3}$ et $\langle,\rangle^{\otimes 3}$ sont définis positifs, on déduit de (5) que lorsque $f \in$ Prim est telle que $L(f, 1)=0$, alors $\left(\mathbf{1}_{f} \otimes \mathbf{1}_{h} \otimes \mathbf{1}_{h}\right)\left(\Delta_{3}\right)=0(h \in$ Prim $)$. Par ailleurs, rappelons que l'idéal $I_{e}$ de $\mathbb{T}$ est l'annulateur de l'ensemble des formes paraboliques $f$ pour lesquelles $L(f, 1) \neq 0$ (voir [Merel 1996]). On en déduit que, lorsque $f \in$ Prim est telle que $L(f, 1) \neq 0$, le vecteur propre associé $a_{f}$ est dans $\mathscr{P}_{\overline{\mathbb{Q}}}^{0}\left[I_{e}\right]$ et donc $\left(\mathbf{1}_{f} \otimes \mathbf{1}_{h} \otimes \mathbf{1}_{h}\right)\left(\Delta_{3}\right) \in \mathscr{P}^{0}\left[I_{e}\right]_{\mathbb{Q}} \otimes_{\mathbb{Q}} \mathscr{P}_{\mathbb{Q}}^{0} \otimes_{\mathbb{Q}} \mathscr{P}_{\mathbb{Q}}^{0}(h \in \operatorname{Prim})$.

On déduit immédiatement du Théorème 2.1 le corollaire suivant :

Corollaire 2.3. Pour toute forme $\mathbb{Q}$-linéaire $\phi$ sur $\mathscr{P}_{\mathbb{Q}_{\mathbb{Q}}}^{0} \otimes_{\mathbb{T}_{\mathbb{Q}}} \mathscr{P}_{\mathbb{Q}}^{0}$, on a $\left(1 \otimes_{\mathbb{Q}} \phi\right)\left(\bar{\Delta}_{3}^{0}\right) \in$ $\mathscr{P}^{0}\left[I_{e}\right]_{\mathbb{Q}}$.

Par définition de $\theta$, on a

$$
y_{m}=\left(1 \otimes\left(a_{m} \circ \theta\right)\right)\left(\bar{\Delta}_{3}\right) \quad \text { et } \quad y_{m}^{0}=\left(1 \otimes_{\mathbb{Q}}\left(a_{m} \circ \theta^{0}\right)\right)\left(\bar{\Delta}_{3}^{0}\right) .
$$

Le Théorème 0.1 énoncé dans l'introduction se déduit alors du corollaire 2.3 appliqué à $\phi=a_{m} \circ \theta^{0}$.

Remarque 2.4. Toute forme linéaire sur $\mathscr{P}_{\mathbb{Q}}^{0} \otimes_{\mathbb{T}_{\mathbb{Q}}} \mathscr{P}_{\mathbb{Q}}^{0} \mathrm{~s}^{\prime}$ obtient comme combinaison linéaire des formes linéaires $a_{m} \circ \theta^{0}(m \geq 1)$ car $\theta^{0}: \mathscr{P}_{\mathbb{Q}} \otimes_{\mathbb{T}_{\mathbb{Q}}} \mathscr{P}_{\mathbb{Q}}^{0} \rightarrow \mu_{\mathbb{Q}}^{0}$ est un isomorphisme. Il n'est donc pas restrictif de considérer les combinaisons linéaires d'éléments $y_{m}^{0}$ comme nous le faisons par la suite. 


\section{Les éléments $y_{m}$}

3A. Comparaison avec les éléments de Gross. Soit $D>0$. Notons $O_{-D}$ l'ordre quadratique de discriminant $-D$ s'il existe et $u(-D)$ l'ordre de $\mathbb{O}_{-D}^{*} /\langle \pm 1\rangle$. Pour $i \in\{0, \ldots, g\}$, les anneaux $R_{i}=$ End $E_{i}, i \in\{0, \ldots, g\}$, sont des ordres maximaux de l'algèbre de quaternions $\mathscr{B}$ sur $\mathbb{Q}$ ramifiée en $p$ et $\infty$; on note $h_{i}(-D)$ le nombre de plongements optimaux de $\mathrm{O}_{-D}$ dans $R_{i}$ modulo conjugaison par $R_{i}^{*}$. Le $D$-ième élément de Gross $^{4}$ est défini par

$$
\gamma_{D}=\frac{1}{2 u(-D)} \sum_{i=0}^{g} h_{i}(-D) x_{i} \in \mathscr{P}_{\mathbb{Z}[1 / 6]} .
$$

On a $\gamma_{D}=0$ si $-D$ n'est pas un discriminant quadratique imaginaire ou bien si $p$ est décomposé dans $\mathbb{Q}(\sqrt{-D})$ (en effet, dans ce dernier cas, $O_{-D}$ ne se plonge pas dans l'algèbre de quaternions ramifiée en $p$ et l'infini).

Démonstration de la proposition 0.2. Soit $m \geq 1$ un entier. L'opérateur $T_{m}$ agissant sur $x_{i}$ par la transposée de la matrice de Brandt $B(m)$, on a $y_{m}=\sum_{i=0}^{g} B_{i, i}(m) x_{i}$. Notons $N$ la norme réduite et tr la trace sur $\mathscr{B}$. On a

où

$$
B_{i, i}(m)=\frac{1}{2 w_{i}} \operatorname{Card}\left\{b \in R_{i} ; N(b)=m\right\}=\frac{1}{2 w_{i}} \sum_{\substack{s \in \mathbb{Z} \\ s^{2} \leq 4 m}} \operatorname{Card}\left(A_{i}(s, m)\right)
$$

$$
A_{i}(s, m)=\left\{b \in R_{i} ; N(b)=m, \operatorname{tr}(b)=s\right\} .
$$

Posons $D=4 m-s^{2}$. Lorsque $D=0$, ce qui est possible si et seulement si $m$ est un carré, $A_{i}(s, m)$ n'a qu'un seul élément. Considérons maintenant le cas où $D>0$. Les éléments de $A_{i}(s, m)$ sont en bijection avec les plongements de $\mathrm{O}_{-} D$ dans $R_{i}$. Pour chaque tel plongement $f$ il existe un unique ordre $\mathrm{O}_{-d}$ contenant $\mathcal{O}_{-D}\left(D=d r^{2}\right.$ pour un certain $\left.r\right)$ tel que $f$ s'étende en un plongement optimal de $0_{-d}$ dans $R_{i}$. On a donc une partition

$$
A_{i}(s, m)=\bigsqcup_{\substack{d \in \mathbb{N} ; \exists r \in \mathbb{N} ; \\ d r^{2}=D}} A_{i}(s, m)_{d}
$$

où les éléments de $A_{i}(s, m)_{d} \subset A_{i}(s, m)$ correspondent aux plongements de $\mathbb{O}_{-} D$ dans $R_{i}$ qui s'étendent en un plongement optimal de $0_{-d}$ dans $R_{i}$. Comme

$$
\operatorname{Card}\left(A_{i}(s, m)_{d}\right)=h_{i}(-d)\left|R_{i}^{\times} / \mathcal{O}_{-d}^{\times}\right|=w_{i} h_{i}(-d) / u(-d),
$$

\footnotetext{
${ }^{4}$ Cet élément, introduit par Gross, est noté $e_{D}$ dans [Gross 1987] et [Parent 2005].
} 
on a finalement $\operatorname{Card}\left(A_{i}(s, m)\right)=w_{i} \sum_{\substack{d \in \mathbb{N} ; \exists r \in \mathbb{N} \\ d r^{2}=D}} \frac{h_{i}(-d)}{u(-d)}$. Par conséquent, pour tout

$$
\begin{aligned}
y_{m} & =\sum_{4 m=s^{2}} \sum_{i=0}^{g} \frac{x_{i}}{2 w_{i}}+\sum_{i=0}^{g} \sum_{\substack{(s, d) \in \mathbb{Z} \times \mathbb{N} \\
\exists r>0 ; 4 m-s^{2}=d r^{2}>0}} \frac{h_{i}(-d)}{2 u(-d)} x_{i} \\
& =\epsilon(m) a_{E}+\sum_{\substack{(s, d) \in \mathbb{Z} \times \mathbb{N} \\
4 m-s^{2}=d r^{2}>0}} \sum_{i=0}^{g} \frac{h_{i}(-d)}{2 u(-d)} x_{i} .
\end{aligned}
$$

Remarque 3.1. Le raisonnement précédent est celui qui donne la formule d'Eichler pour la trace de $T_{m}$ (voir [Eichler 1955] ou [Gross 1987]). On retrouve cette formule en identifiant les degrés de chacun des membres de l'égalité de la proposition 0.2.

Remarque 3.2. La formule de Gross [1987, corollaire 11.6] montre que pour tout entier $d<0$ premier à $p$, l'élément $\gamma_{d}^{0}$ est dans $\mathscr{P}^{0}\left[I_{e}\right]_{\mathbb{Q}}$ (voir par exemple [Parent 2005]). La proposition 0.2 donne alors une nouvelle preuve du Théorème 0.1 dans le cas particulier où $m$ est tel que tout entier $d>0$ tel que $4 m-s^{2}=d r^{2}$ soit premier à $p$.

À titre d'exemple, voici la décomposition de $y_{m}$ comme combinaison linéaire d'éléments de Gross pour $m \leq 13$ :

$$
\begin{aligned}
y_{1} & =a_{E}+2 \gamma_{3}+\gamma_{4}, \\
y_{2} & =2 \gamma_{4}+2 \gamma_{7}+\gamma_{8}, \\
y_{3} & =3 \gamma_{3}+2 \gamma_{8}+2 \gamma_{11}+\gamma_{12}, \\
y_{4} & =a_{E}+2 \gamma_{3}+\gamma_{4}+2 \gamma_{7}+2 \gamma_{12}+2 \gamma_{15}+\gamma_{16}, \\
y_{5} & =4 \gamma_{4}+2 \gamma_{11}+2 \gamma_{16}+2 \gamma_{19}+\gamma_{20}, \\
y_{6} & =2 \gamma_{8}+2 \gamma_{15}+2 \gamma_{20}+2 \gamma_{23}+\gamma_{24}, \\
y_{7} & =6 \gamma_{3}+\gamma_{7}+2 \gamma_{12}+2 \gamma_{19}+2 \gamma_{24}+2 \gamma_{27}+\gamma_{28}, \\
y_{8} & =2 \gamma_{4}+4 \gamma_{7}+\gamma_{8}+2 \gamma_{16}+2 \gamma_{23}+2 \gamma_{28}+2 \gamma_{31}+\gamma_{32}, \\
y_{9} & =a_{E}+2 \gamma_{3}+\gamma_{4}+2 \gamma_{8}+2 \gamma_{11}+2 \gamma_{20}+2 \gamma_{27}+2 \gamma_{32}+2 \gamma_{35}+\gamma_{36}, \\
y_{10} & =4 \gamma_{4}+2 \gamma_{15}+2 \gamma_{24}+2 \gamma_{31}+2 \gamma_{36}+2 \gamma_{39}+\gamma_{40}, \\
y_{11} & =2 \gamma_{7}+2 \gamma_{8}+\gamma_{11}+2 \gamma_{19}+2 \gamma_{28}+2 \gamma_{35}+2 \gamma_{40}+2 \gamma_{43}+\gamma_{44}, \\
y_{12} & =3 \gamma_{3}+2 \gamma_{8}+2 \gamma_{11}+3 \gamma_{12}+2 \gamma_{23}+2 \gamma_{32}+2 \gamma_{39}+2 \gamma_{44}+2 \gamma_{47}+\gamma_{48}, \\
y_{13} & =6 \gamma_{3}+4 \gamma_{4}+2 \gamma_{12}+2 \gamma_{16}+2 \gamma_{27}+2 \gamma_{36}+2 \gamma_{43}+2 \gamma_{48}+2 \gamma_{51}+\gamma_{52} .
\end{aligned}
$$

3B. Espace vectoriel et module de Hecke engendrés par ces éléments. Nous démontrons dans ce paragraphe les propositions 0.3 et 0.4 ainsi que le corollaire 
0.5. Faisons tout d'abord quelques observations. A une forme linéaire $\phi$ sur $\mathscr{P}_{\mathbb{Q}}$, associons la forme modulaire $\mathbf{g}_{\phi}=\left(\phi \otimes_{\mathbb{Q}} \theta^{0}\right)\left(\bar{\Delta}_{3}^{0}\right) \in \mathcal{M}_{\mathbb{Q}}^{0}$. Remarquons que $\mathbf{g}_{\phi}$ a pour $q$-développement

$$
\sum_{m \geq 1} \phi\left(y_{m}^{0}\right) q^{m}
$$

De plus, puisque $\phi \otimes_{\overline{\mathbb{Q}}} \theta^{0}$ est un homomorphisme de $\mathbb{T}_{\overline{\mathbb{Q}}} \otimes_{\overline{\mathbb{Q}}} \mathbb{T}_{\overline{\mathbb{Q}}}$-modules, on a

$$
\mathbf{1}_{h} \mathbf{g}_{\phi}=\left(\phi \otimes_{\overline{\mathbb{Q}}} \theta^{0}\right)\left(\sum_{g \in \text { Prim }}\left(\mathbf{1}_{g} \otimes_{\overline{\mathbb{Q}}} \mathbf{1}_{h}\right)\left(\bar{\Delta}_{3}\right)\right) \quad(h \in \text { Prim }) .
$$

Démonstration de la proposition 0.3. Il suffit de montrer que toute forme linéaire sur l'espace vectoriel engendré par $\left(y_{m}^{0}\right)_{m \geq 1}$ et qui s'annule en $y_{1}^{0}, \ldots, y_{g+1}^{0}$ est nulle. Soit $\phi$ une telle forme linéaire. La forme différentielle $\omega_{\phi}$ de $X_{0}(p)$ associée à $\mathbf{g}_{\phi}$ a pour $q$-développement

$$
\sum_{m \geq 1} \phi\left(y_{m}^{0}\right) q^{m} \frac{d q}{q} .
$$

Si $\phi\left(y_{1}^{0}\right)=0, \ldots, \phi\left(y_{g+1}^{0}\right)=0$, la forme différentielle holomorphe $\omega_{\phi}$ a un zéro d'ordre $g$ en l'infini. L'infini n'étant pas un point de Weierstrass de $X_{0}(p)$ (voir [Ogg 1978]), on en déduit que $\omega_{\phi}$ est nulle, d'où la proposition.

Pour démontrer la proposition 0.4 on a encore besoin de deux lemmes :

Lemme 3.3. Soient $f$ et $h$ deux formes primitives. Les assertions suivantes sont équivalentes:

$$
\text { a) } L(f, 1) L\left(f \otimes \operatorname{Sym}^{2} h, 2\right)=0 ; \quad \text { b) } \mathbf{1}_{h \cdot} \cdot \mathbf{g}_{\left\langle., a_{f}\right\rangle}=0 .
$$

Démonstration. D'après (9), on a

$$
\mathbf{1}_{h} \mathbf{g}_{\left\langle., a_{f}\right\rangle}=\left(\left\langle., a_{f}\right\rangle \otimes_{\overline{\mathbb{Q}}} \theta^{0}\right)\left(\sum_{g \in \text { Prim }}\left(\mathbf{1}_{g} \otimes_{\overline{\mathbb{Q}}} \mathbf{1}_{h}\right)\left(\bar{\Delta}_{3}\right)\right) .
$$

Puisque $\left\langle a_{g}, a_{f}\right\rangle=0$ si $g \neq f$, on obtient

$$
\mathbf{1}_{h} \mathbf{g}_{\left\langle., a_{f}\right\rangle}=\left(\left\langle., a_{f}\right\rangle \otimes_{\overline{\mathbb{Q}}} \theta^{0}\right)\left(\mathbf{1}_{f} \otimes_{\overline{\mathbb{Q}}} \mathbf{1}_{h}\right)\left(\bar{\Delta}_{3}\right) .
$$

Or l'application

$$
\left\langle., a_{f}\right\rangle \otimes_{\overline{\mathbb{Q}}} \theta^{0}: \mathscr{P}_{\overline{\mathbb{Q}}}^{f} \otimes_{\overline{\mathbb{Q}}}\left(\mathscr{P}_{\overline{\mathbb{Q}}}^{0} \otimes_{\mathbb{T}_{\overline{\mathbb{Q}}}} \mathscr{P}_{\overline{\mathbb{Q}}}^{0}\right) \rightarrow M_{\overline{\mathbb{Q}}}^{0}
$$

est injective car $\theta^{0}$ est un isomorphisme de $\mathbb{T}_{\mathbb{Q}}$-modules libres (voir fin du Section 1A). Par conséquent $\mathbf{1}_{h} \mathbf{g}_{\left\langle., a_{f}\right\rangle}=0$ si et seulement si $\left(\mathbf{1}_{f} \otimes_{\overline{\mathbb{Q}}} \mathbf{1}_{h}\right)\left(\bar{\Delta}_{3}\right)=0$ i.e. si et seulement si $\left(\mathbf{1}_{f} \otimes_{\overline{\mathbb{Q}}} \mathbf{1}_{h} \otimes_{\overline{\mathbb{Q}}} \mathbf{1}_{h}\right)\left(\Delta_{3}\right)=0$. D'après (5), ceci est équivalent à l'assertion $a$ ). 
Lemme 3.4. Les conditions suivantes sont équivalentes:

i) le $\mathbb{T}_{\mathbb{Q}}$-module y engendré par $\left\{y_{m}^{0}, m \geq 1\right\}$ est égal à $\mathscr{P}^{0}\left[I_{e}\right]_{\mathbb{Q}}$;

ii) pour toute forme primitive $f$ de poids 2 pour $\Gamma_{0}(p)$ telle que $L(f, 1) \neq$ 0 , il existe une forme primitive $h$ de poids 2 pour $\Gamma_{0}(p)$ telle que $L(f \otimes$ $\left.\mathrm{Sym}^{2} h, 2\right) \neq 0$.

Démonstration. Les espaces $\mathbb{T}_{\overline{\mathbb{Q}}}$-propres de $\mathscr{P}_{\overline{\mathbb{Q}}}^{0}$ étant des $\overline{\mathbb{Q}}$-droites, on a $\mathscr{Y}=$ $\mathscr{P}^{0}\left[I_{e}\right]_{\mathbb{Q}}$ si et seulement si pour tout $f \in$ Prim tel que $L(f, 1) \neq 0$, il existe $m \geq 1$ tel que $\mathbf{1}_{f} y_{m} \neq 0$. Par ailleurs, d'après le lemme 3.3, la condition ii) du lemme est vérifiée si et seulement si pour tout $f \in$ Prim tel que $L(f, 1) \neq 0$, on a $\mathbf{g}_{\left\langle., a_{f}\right\rangle} \neq 0$ (car il existe alors $h$ telle que $\mathbf{1}_{h} \mathbf{g}_{\left\langle., a_{f}\right\rangle} \neq 0$ ). Puisque $\mathbf{g}_{\left\langle., a_{f}\right\rangle}$ a pour $q$-développement $\sum_{m \geq 1}\left\langle y_{m}, a_{f}\right\rangle q^{m}$ (voir (8)), on en déduit l'équivalence des assertions i) et ii).

Démonstration de la proposition 0.4. D'après les travaux de Böcherer et SchulzePillot [1999] améliorés par Arakawa et Böcherer [2003, théorème 5.3], la condition ii) du lemme ci-dessus est toujours satisfaite.

Démonstration $d u$ corollaire 0.5 . Soit $f \in$ Prim telle que $L(f, 1) \neq 0$. D'après les propositions 0.3 et 0.4 , il existe alors $1 \leq m \leq g+1$ tel que $\mathbf{1}_{f} y_{m} \neq 0$. Par conséquent, il existe $D \leq 4 g+4$ tel que $\mathbf{1}_{f} \gamma_{D} \neq 0$. On a $(D, p)=1$ car $D \leq 4 g+4$. Soit $-d$ le discriminant fondamental de $\mathbb{Q}(\sqrt{-D})$. En vertu des relations de norme [Bertolini et Darmon 1996] 2.4, on a $\gamma_{D} \in \widetilde{\mathbb{T}} \cdot \gamma_{d}$. On en déduit que $\mathbf{1}_{f} \gamma_{d} \neq 0$ et $L\left(f \otimes \varepsilon_{d}, 1\right) \neq 0$ d'après la formule de Gross [1987, corollaire 11.6] généralisée par Zhang [2001, théorème 1.3.2].

\section{Points rationnels de courbes modulaires}

4A. Une question théorique. On suppose désormais $p \geq 11$. Soit $\mathbb{Z}_{(p)}$ le localisé de $\mathbb{Z}$ en $p$. Soit $j \in \mathbb{P}^{1}\left(\overline{\mathbb{F}}_{p}\right)$ non supersingulier. On étend $\iota_{j}$ à $\mathscr{P}_{\mathbb{Z}_{(p)}}$ en posant $\iota_{j}(1 / a)=a^{-1}$. Supposons vérifiée la condition :

(H) Pour tout $j \in \mathbb{F}_{p}$ ordinaire, il existe $m \geq 1$ tel que $\iota_{j}\left(y_{m}^{0}\right) \neq 0$.

D'après le Théorème 0.1 et le critère $(\mathrm{C})$ énoncé dans l'introduction, on a alors $X_{0}^{+}\left(p^{r}\right)(\mathbb{Q})$ trivial. Actuellement, on ne sait pas si pour $p$ assez grand (par exemple $p>37)$, l'hypothèse $(\mathrm{H})$ est vérifiée. Dans l'espoir d'obtenir un résultat sans les contraintes de congruence du Théorème 0.6 , remarquons que l'on peut reformuler cette condition. On a $\left(1 \otimes \theta^{0}\right)\left(\bar{\Delta}_{3}^{0}\right) \in \mathscr{P}_{\mathbb{Z}[1 / n]}^{0} \otimes M^{0}$. Considérons la forme parabolique à coefficients dans $\overline{\mathbb{F}}_{p}\left(\right.$ i.e. l'élément de $\left.\overline{\mathbb{F}}_{p} \otimes \mathcal{M}^{0}\right)$ définie par

$$
\mathbf{g}_{j}=\left(\iota_{j} \otimes \theta^{0}\right)\left(\bar{\Delta}_{3}^{0}\right) \in \mathcal{M}_{\overline{\mathbb{F}}_{p}}^{0} .
$$

Cette forme modulaire a pour $q$-développement $\sum_{m \geq 1} \iota_{j}\left(y_{m}^{0}\right) q^{m}$. L'hypothèse $(\mathrm{H})$ est donc équivalente à : 
$\left(\mathrm{H}^{\prime}\right) \quad$ Pour tout $j \in \mathbb{F}_{p}$ ordinaire, on a $\mathbf{g}_{j} \neq 0$.

4B. Calculs pratiques : démonstration du Théorème 0.6. Soit $j \in \mathbb{F}_{p}$ non supersingulier. On a

$$
\iota_{j}\left(y_{m}^{0}\right)=\sum_{i=0}^{g} \frac{B_{i, i}(m)}{j-j_{i}}+12 \operatorname{tr}(B(m)) \sum_{i=0}^{g} \frac{1}{w_{i}\left(j-j_{i}\right)} \in \overline{\mathbb{F}}_{p} .
$$

En effet,

$$
y_{m}^{0}=y_{m}-\frac{12}{p-1} \operatorname{deg}\left(y_{m}\right) a_{E} \quad \text { et } \quad \iota_{j}\left(a_{E}\right)=\sum_{i=0}^{g} \frac{1}{w_{i}\left(j-j_{i}\right)} .
$$

Le membre de droite de cette dernière égalité n'étant pas facile à calculer, nous introduisons

$$
y_{k, m}=\operatorname{tr} B(m) y_{k}-\operatorname{tr} B(k) y_{m} \in \mathscr{P}^{0}\left[I_{e}\right]_{\mathbb{Q}} \quad(0<k<m)
$$

et calculons $\iota_{j}\left(y_{k, m}\right)$ pour $k, m$ dans $\{2,3,5,6,7\}$.

Lorsque $(m, p)=1$, l'entier $B_{i, i}(m)$ est la multiplicité de $j_{i}$ comme racine du polynôme modulaire $\phi_{m}(X, X)$ dans $\overline{\mathbb{F}}_{p}$ (voir [Igusa 1959] ou [Lang 1987, 5.3 théorème 5]). Les polynômes modulaires $\phi_{m}(X, X)$ pour $m \in\{2,3,5,6,7\}$, donnés par Magma, sont :

$$
\begin{aligned}
& \phi_{2}(X, X)=-(X-1728)(X+3375)^{2}(X-8000), \\
& \phi_{3}(X, X)=-X(X-54000)(X+32768)^{2}(X-8000)^{2}, \\
& \begin{aligned}
& \phi_{5}(X, X)=-(X-1728)^{2}(X-287496)^{2}(X+32768)^{2}(X+884736)^{2} P_{1}, \\
& \phi_{6}(X, X)=(X-8000)^{2} P_{1}^{2} P_{2} P_{3}^{2} P_{4}^{2}, \\
& \phi_{7}(X, X)=-(X+3375)(X-16581375) X^{2}(X-54000)^{2} \\
& \times(X+12288000)^{2}(X+884736)^{2} P_{2}^{2},
\end{aligned}
\end{aligned}
$$

où

$$
\begin{aligned}
& P_{1}=X^{2}-1264000 X-681472000, \\
& P_{2}=X^{2}-4834944 X+14670139392, \\
& P_{3}=X^{2}+191025 X-121287375, \\
& P_{4}=X^{3}+3491750 X^{2}-5151296875 X+12771880859375 .
\end{aligned}
$$

Comme $m$ n'est pas carré, les racines de $\phi_{m}(X, X)$ dans $\mathbb{C}$ sont les invariants $j(\tau)$ de courbes elliptiques à multiplication complexe par un ordre quadratique imaginaire $\mathbb{Z}[\tau]$. Les ordres quadratiques associés aux invariants racines des facteurs de degré 1 sont donnés dans la littérature (voir par exemple [Cohen 1993, 7.2.3]). En 
déterminant les ordres quadratiques imaginaires possédant un élément de norme 5,6 et 7 , on trouve une racine $\alpha_{i}$ du facteur $P_{i}$ de degré 2 pour $i \in\{1, \ldots, 4\}$. On obtient ainsi les valeurs de $B_{i, i}(m)(m \in\{2,3,5,6,7\})$ données dans la table ci-dessous où $a, b, c, d, e, f, g, h, v, w \in\{0,1\}$ sont par définition égaux à 1 si et seulement si $j(\tau)$ est supersingulier modulo $p$, c'est-à-dire si $p$ est inerte ou ramifié dans $\mathbb{Q}(\tau)$.

On supposera désormais que $p>173$. Dans ce cas, les invariants apparaissant dans cette table sont tous distincts.

L'égalité $a=c$ équivaut à $h=d$ et $a=d$ équivaut à $v=g$. On fait parcourir au 8-uplet $(a, b, c, d, e, f, g, w)$ les différentes valeurs possibles. Pour tous les 8-uplets distincts de ceux énumérés dans la Table 2 , les fractions $\iota_{j}\left(y_{k, m}\right)$, où $k, m \in\{2,3,5,6,7\}$, ne s'annulent pas simultanément. La table résume les résultats obtenus pour tous les 8 -uplets posant un problème. Lorsque $\iota_{j}\left(y_{k, m}\right)$ n'est pas une fraction identiquement nulle, on note $n_{k, m}$ le degré de son numérateur. Lorsque $n_{k, m}=2, \iota_{j}\left(y_{k, m}\right)$ a un zéro dans $\mathbb{F}_{p}$ si et seulement si le discriminant $d_{k, m}$ de son numérateur (dans $\mathbb{Z}$ ) est un carré modulo $p$.

\begin{tabular}{|c|c|c|c|c|c|c|c|c|}
\hline$j_{i}$ & $\tau$ & $D_{\tau}$ & & $B_{i}(2)$ & $B_{i}(3)$ & $B_{i}(5)$ & $B_{i}(6)$ & $B_{i}(7)$ \\
\hline 1728 & $\sqrt{-1}$ & 4 & $a$ & 1 & & 2 & & \\
\hline 287496 & $2 \sqrt{-1}$ & 4 & $a$ & & & 2 & & \\
\hline-3375 & $\frac{1}{2}(1+\sqrt{-7})$ & 7 & $b$ & 2 & & & & 1 \\
\hline 16581375 & $\sqrt{-7}$ & 7 & $b$ & & & & & 1 \\
\hline 8000 & $\sqrt{-2}$ & 8 & $c$ & 1 & 2 & & 2 & \\
\hline 0 & $\frac{1}{2}(1+\sqrt{-3})$ & 3 & $d$ & & 1 & & & 2 \\
\hline 54000 & $\sqrt{-3}$ & 3 & $d$ & & 1 & & & 2 \\
\hline-12288000 & $\frac{1}{2}(1+3 \sqrt{-3})$ & 3 & $d$ & & & & & 2 \\
\hline-32768 & $\frac{1}{2}(1+\sqrt{-11})$ & 11 & $e$ & & 2 & 2 & & \\
\hline-884736 & $\frac{1}{2}(1+\sqrt{-19})$ & 19 & $f$ & & & 2 & & 2 \\
\hline$\alpha_{1}$ & $\sqrt{-5}$ & 20 & $g$ & & & 1 & 2 & \\
\hline$\alpha_{2}$ & $\sqrt{-6}$ & 24 & $h$ & & & & 1 & 2 \\
\hline$\alpha_{3}$ & $\frac{1}{2}(1+\sqrt{-15})$ & 15 & $v$ & & & & 2 & \\
\hline$\alpha_{4}$ & $\frac{1}{2}(1+\sqrt{-23})$ & 23 & $w$ & & & & 2 & \\
\hline
\end{tabular}

Table 1. Valeurs de $B_{i}(m)=B_{i, i}(m), m \in\{2,3,5,6,7\}$. 


\begin{tabular}{|c|c|c|c|}
\hline$a b c c d$ & $e f$ & $g \quad v$ & Résultat \\
\hline $\begin{array}{llllll}0 & 0 & 1 & 0\end{array}$ & $0 \quad 0$ & 0 & voir texte ci-dessous \\
\hline \multirow[t]{3}{*}{$\begin{array}{lllll}0 & 0 & 0 & 0\end{array}$} & $0 \quad 0$ & $\begin{array}{l}0 \\
1 \\
1\end{array}$ & $\begin{array}{l}\iota_{j}\left(y_{k, m}\right)=0(k, m \in\{2,3,5,6,7\}) \\
n_{5,6}=2, d_{5,6}=2^{6} .5^{6} .11^{5} .13^{4} .37^{2} .59 .71, \\
\iota_{j}\left(y_{k, m}\right)=0(k, m) \neq(5,6) \\
n_{5,6}=5, \quad \iota_{j}\left(y_{k, m}\right)=0(k, m) \neq(5,6)\end{array}$ \\
\hline & $\begin{array}{ll}0 & 1\end{array}$ & $\begin{array}{l}0 \\
0\end{array}$ & $\begin{array}{l}\iota_{j}\left(y_{k, m}\right)=0(k, m \in\{2,3,5,6,7\}) \\
\iota_{j}\left(y_{5,6}\right)=\iota_{j}\left(y_{6,7}\right) n_{5,6}=2, \\
d_{5,6}=2^{6} \cdot 5^{7} \cdot 7^{4} \cdot 31.36319 \cdot p_{0} \\
\iota_{j}\left(y_{k, m}\right)=0 \mathrm{si}(k, m) \neq(5,6),(6,7)\end{array}$ \\
\hline & 10 & $\begin{array}{l}0 \\
0\end{array}$ & $\begin{array}{l}\iota_{j}\left(y_{k, m}\right)=0(k, m \in\{2,3,5,6,7\}) \\
\iota_{j}\left(y_{3,6}\right)=\iota_{j}\left(y_{5,6}\right), n_{5,6}=2, \\
d_{5,6}=2^{6} \cdot 3 \cdot 5^{7} \cdot 7^{8} \cdot 11^{2} \cdot 17^{2} \cdot 19^{2} \cdot 797 \\
\iota_{j}\left(y_{k, m}\right)=0 \mathrm{si}(k, m) \neq(3,6),(5,6)\end{array}$ \\
\hline
\end{tabular}

Table 2. Résultats des calculs pous les cas exceptionnels. Le symbole $*$ signifie que le coefficient peut prendre indifféremment la valeur 0 ou 1 . On rappelle que $p_{0}=45321935159$.

À titre d'exemple, lorsque $(a, b, c, d, e, f, g, w)=(0,0,1,0,0,0,0,0)$, on obtient

$$
\iota_{j}\left(y_{2,3}\right)=\iota_{j}\left(y_{2,5}\right)=\iota_{j}\left(y_{3,5}\right)=\iota_{j}\left(y_{5,6}\right)=\iota_{j}\left(y_{5,7}\right)=0
$$

et $\iota_{j}\left(y_{2,6}\right), \iota_{j}\left(y_{3,6}\right), \iota_{j}\left(y_{2,7}\right), \iota_{j}\left(y_{3,7}\right)$ et $\iota_{j}\left(y_{6,7}\right)$ sont, à multiplication par une puissance de 2 près, égaux à

$$
Q(j)=\frac{2}{j-8000}-\frac{P_{2}^{\prime}(j)}{P_{2}(j)}=\frac{-2^{11}\left(13.181 j+2^{6} \cdot 3^{3} \cdot 7.13 .29\right)}{(j-8000) P_{2}(j)}
$$

qui s'annule en $j_{0} \equiv-(181)^{-1} \cdot 2^{6} \cdot 3^{3} \cdot 7 \cdot 29 \bmod p$.

Posons $\mathscr{\Re}$ l'ensemble des nombres premiers $p$ qui sont simultanément un carré modulo 3, 4 et 7 et qui vérifient l'une des conditions suivantes :

i) $p$ carré modulo $5,11,19$, et 23 et non carré modulo 8 ,

ii) $p$ est un carré modulo $5,8,11$ et 19 ;

iii) $p$ carré modulo 8,11 et 19 , non carré modulo 5, 23;

iv) $p$ carré modulo $8,11,19,23,59,71$, non carré modulo 5 ;

v) $p$ carré modulo $8,11,19,23$, non carré modulo 5, 59, 71; 
vi) $p$ carré modulo $5,8,11,23$, non carré modulo 19 ;

vii) $p$ carré modulo 5, 8, 11, non carré modulo 19,23 et $\left(\frac{p}{31}\right)\left(\frac{p}{36319}\right)\left(\frac{p}{p_{0}}\right)=1$; viii) $p$ carré modulo $5,8,19,23$, non carré modulo 11 ;

ix) $p$ carré modulo 5, 8, 19, 797, non carré modulo 11, 23.

Lemme 4.1. Si $p>173, p \neq 797,36319$, $p_{0}$ et $p \notin \mathscr{B}$, alors pour tout $j \in \mathbb{F}_{p}$ non supersingulier, il existe $(k, m) \in\{2,3,5,6,7\}^{2}$ tel que $\iota_{j}\left(y_{k, m}\right) \neq 0$ et par conséquent $X_{0}^{+}\left(p^{r}\right)(\mathbb{Q})$ est trivial.

Démonstration. On vérifie aisément que lorsque $p$ est comme dans l'énoncé et $j \in \mathbb{F}_{p}$, nous ne sommes pas dans l'un des cas énumérés dans la Table 2 et donc il y a une fraction non nulle parmi $\iota_{j}\left(y_{k, m}\right),(k, m) \in\{2,3,5,6,7\}^{2}$.

Le lemme 4.1 et le théorème 1.1 de [Parent 2005] entraînent alors le Théorème 0.6. En effet, l'ensemble $\mathscr{C}$ du Théorème 0.6 est égal à $\mathscr{A} \cap \mathscr{B}$ et $797,36319, p_{0}$ ne sont pas dans $\mathscr{A}$ (ici $\mathscr{A}$ est l'ensemble du théorème 1.1 de [Parent 2005]). Le cas des nombres premiers $p=11$ et $17 \leq p \leq 173$ a été traité par Parent [2005, p. 8, preuve du théorème 1.1].

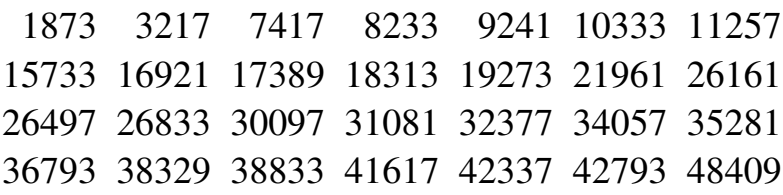

Table 3. Nombres premiers $p \leq 50000$ dans l'ensemble $\mathscr{C}$.

\section{Remerciements}

Je tiens ici à remercier L. Merel, P. Parent ainsi que le referee pour leurs remarques durant l'élaboration de cet article.

\section{References}

[Arakawa et Böcherer 2003] T. Arakawa et S. Böcherer, "Vanishing of certain spaces of elliptic modular forms and some applications", J. Reine Angew. Math. 559 (2003), 25-51. MR 2004m:11069 Zbl 1043.11040

[Bertolini et Darmon 1996] M. Bertolini et H. Darmon, "Heegner points on Mumford-Tate curves", Invent. Math. 126:3 (1996), 413-456. MR 97k:11100 Zbl 0882.11034

[Bertolini et Darmon 1997] M. Bertolini et H. Darmon, "A rigid analytic Gross-Zagier formula and arithmetic applications”, Ann. of Math. (2) 146:1 (1997), 111-147. MR 99f:11079 Zbl 1029.11027

[Böcherer et Schulze-Pillot 1999] S. Böcherer et R. Schulze-Pillot, "Squares of automorphic forms on quaternion algebras and central values of $L$-functions of modular forms", J. Number Theory 76:2 (1999), 194-205. MR 2000h:11046 Zbl 0940.11023 
[Cohen 1993] H. Cohen, A course in computational algebraic number theory, Graduate Texts in Mathematics 138, Springer, Berlin, 1993. MR 94i:11105 Zbl 0786.11071

[Eichler 1955] M. Eichler, “Zur Zahlentheorie der Quaternionen-Algebren”, J. Reine Angew. Math. 195 (1955), 127-151. MR 18,297c Zbl 0068.03303

[Emerton 2002] M. Emerton, "Supersingular elliptic curves, theta series and weight two modular forms”, J. Amer. Math. Soc. 15:3 (2002), 671-714. MR 2003b:11038 Zbl 01739913

[Gross 1987] B. H. Gross, "Heights and the special values of $L$-series", pp. 115-187 dans Number theory (Montreal, 1985), édité par H. Kisilevsky et J. Labute, CMS Conf. Proc. 7, Amer. Math. Soc., Providence, RI, 1987. MR 89c:11082 Zbl 0623.10019

[Gross et Kudla 1992] B. H. Gross et S. S. Kudla, "Heights and the central critical values of triple product L-functions”, Compositio Math. 81:2 (1992), 143-209. MR 93g:11047 Zbl 0807.11027

[Igusa 1959] J.-i. Igusa, "Kroneckerian model of fields of elliptic modular functions", Amer. J. Math. 81 (1959), 561-577. MR 21 \#7214 Zbl 0093.04502

[Illusie 1991] L. Illusie, "Réalisation $l$-adique de l'accouplement de monodromie d'après A. Grothendieck", 196-197 (1991), 27-44. MR 93c:14020 Zbl 0781.14011

[Lang 1987] S. Lang, Elliptic functions, $2^{\mathrm{e}}$ éd., Graduate Texts in Mathematics 112, Springer, New York, 1987. MR 88c:11028 Zbl 0615.14018

[Merel 1996] L. Merel, "Bornes pour la torsion des courbes elliptiques sur les corps de nombres", Invent. Math. 124:1-3 (1996), 437-449. MR 96i:11057 Zbl 0936.11037

[Mestre et Oesterlé $\geq 2008$ ] J.-F. Mestre et J. Oesterlé, "Courbes elliptiques de conducteur premier". Please Non publié.

[Miyake 1989] T. Miyake, Modular forms, Springer, Berlin, 1989. MR 90m:11062 Zbl 0701.11014

[Momose 1984] F. Momose, "Rational points on the modular curves $X_{\text {split }}(p)$ ", Compositio Math. 52:1 (1984), 115-137. MR 86j:11064 Zbl 0574.14023

[Momose 1986] F. Momose, "Rational points on the modular curves $X_{0}^{+}\left(p^{r}\right)$ ", J. Fac. Sci. Univ. Tokyo Sect. IA Math. 33:3 (1986), 441-466. MR 88a:11056 Zbl 0621.14018

[Momose 1987] F. Momose, "Rational points on the modular curves $X_{0}^{+}(N)$ ", J. Math. Soc. Japan 39:2 (1987), 269-286. MR 88h:14031 Zbl 0623.14009

[Ogg 1978] A. P. Ogg, "On the Weierstrass points of $X_{0}(N)$ ", Illinois J. Math. 22:1 (1978), 31-35. MR 57 \#3136 Zbl 0374.14005

[Parent 2005] P. J. R. Parent, "Towards the triviality of $X_{0}^{+}\left(p^{r}\right)(\mathbb{Q})$ for $r>1$ ", Compos. Math. 141:3 (2005), 561-572. MR 2006a:11076 Zbl 02183028

[Raynaud 1991] M. Raynaud, "Jacobienne des courbes modulaires et opérateurs de Hecke”, 196-197 (1991), 9-25. MR 93b:11077 Zbl 0781.14020

[Ribet 1983] K. A. Ribet, "Mod $p$ Hecke operators and congruences between modular forms", Invent. Math. 71:1 (1983), 193-205. MR 84j:10040 Zbl 0508.10018

[Serre 1972] J.-P. Serre, "Propriétés galoisiennes des points d'ordre fini des courbes elliptiques", Invent. Math. 15:4 (1972), 259-331. Réimpression: pp. 1-73 dans ses Euvres, tome 3, Springer, Berlin, 1986. MR 52 \#8126 Zbl 0235.14012

[Serre 1986] J.-P. Serre, "Resume des cours 1975-1976”, pp. 284-291 dans ses Euvres, vol. III, Springer, Berlin, 1986. MR 89h:01109c

[de Shalit 1995] E. de Shalit, "On certain Galois representations related to the modular curve $X_{1}(p)$ ", Compositio Math. 95:1 (1995), 69-100. MR 96i:11063 Zbl 0853.11045

[Zhang 2001] S.-W. Zhang, "Gross-Zagier formula for GL2", Asian J. Math. 5:2 (2001), 183-290. MR 2003k:11101 Zbl 01818531 
Received December 1, 2006. Revised October 15, 2007.

MARUSiA REBOLLEDO

LABORATOIRE DE MATHÉMATIQUeS

UNIVERsité Blaise PASCAL

CAMPUS UNIVERSITAIRE DES CÉZEAUX

63177 AUBIÈRE

FRANCE

Marusia.Rebolledo@math.univ-bpclermont.fr

http://math.univ-bpclermont.fr/ rebolledo/ 